\title{
Experiments on aircraft flight parameter detection by on-skin sensors
}

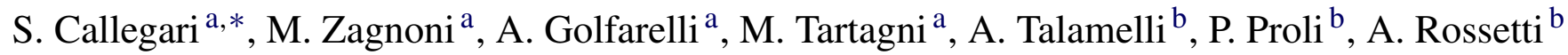 \\ a ARCES and DEIS, University of Bologna, Forlì, Italy \\ b DIEM, University of Bologna, Forli, Italy
}

Received 5 September 2005; received in revised form 24 November 2005; accepted 11 December 2005

Available online 18 January 2006

\begin{abstract}
Air speed and flight attitude angles are fundamental parameters for manual of automatic control of flying bodies. Conventional measurement methods rely on probes (e.g. Pitot tubes or vanes) having a one-to-one correspondence with the physical quantities of interest and requiring specific placements. Here, a novel measurement approach is proposed, relying on indirect measurement and on a plurality of pressure readings made by thin capacitive sensors directly placed on the aircraft skin. A redundant number of probes relaxes the accuracy requirements posed on the individual units and helps achieving fault detection or fault tolerance. A strategy for efficiently processing/combining sensor data is herein presented together with an error propagation analysis, and experimental data.
\end{abstract}

(C) 2005 Elsevier B.V. All rights reserved.

Keywords: Aeronautical measurement system; Flight attitude; Capacitive sensor; Sensor array; Estimation

\section{Introduction}

Modern aircrafts require a vast amount of data coming from on board sensors, either to provide information to the pilot, to implement Fly-by-Wire (FBW) systems, or to achieve automatic flight control as in unmanned air vehicles (UAVs). Specifically, air data is fundamental to infer many high-level flight parameters, including the free-stream air speed and the attitude of flight.

In this regard, it is worth considering that conventional measurement techniques tend to rely on a few specialized, highly accurate elements, in the form of Pitot tubes, electromechanical devices such as vanes, or multifunction units such as selfaligning slot-type sensors [1], etc. In other terms, in conventional systems emphasis is on the transducers, which should make the most direct measurements of the quantities of interest and make them ready available at a good precision. While undoubtedly successful, conventional techniques have a few drawbacks: (1) different probe types may be needed for reading different physical quantities, leading to device proliferation (at times hidden by the use of multi-functional probes [1]); (2) highly accurate probes are generally required, leading to high costs (possibly critical for certain classes of fliers); (3) probe installation may

\footnotetext{
* Corresponding author. Tel.: +39 0543 786927; fax: +390543 786926.

E-mail address: scallegari@arces.unibo.it (S. Callegari).
}

be intrusive, or constrained to very specific locations, or probes may be too bulky for small vehicles; (4) probe placement may be critical with regard to perturbation and wake effects (at times, to the point of requiring booms to bring probes outside the aircraft profile); (5) the use of a small number of sensing devices may make faults extremely critical.

These issues are well recognized and a few attempts have been made to their solution. For instance, multi-hole/multifunctional probes have been introduced to infer a plurality of flight parameters at once [1,2]; techniques have been introduced to get free-stream parameters from measurement made on the air vehicle skin [3]; replicated systems have been introduced to enhance availability [4].

In this paper, a radically different approach is considered and experimented, where the above issues are avoided a-priori by moving the emphasis from the transducers to the signal processing [5]. This is generally sensible, as the processing power made available to designers is rapidly increasing in magnitude and decreasing in cost. Rather than using a few specialized probes, many low cost transducers of the same type and providing homogeneous raw data are employed, showing that efficient signal processing can anyway assure the possibility of inferring a plurality of high-level parameters at an adequate accuracy. The sensors are designed favoring low cost and low intrusivity over other merit factors. Thanks to their large number, the system can be made tolerant of faults. Eventually, sensor placement con- 


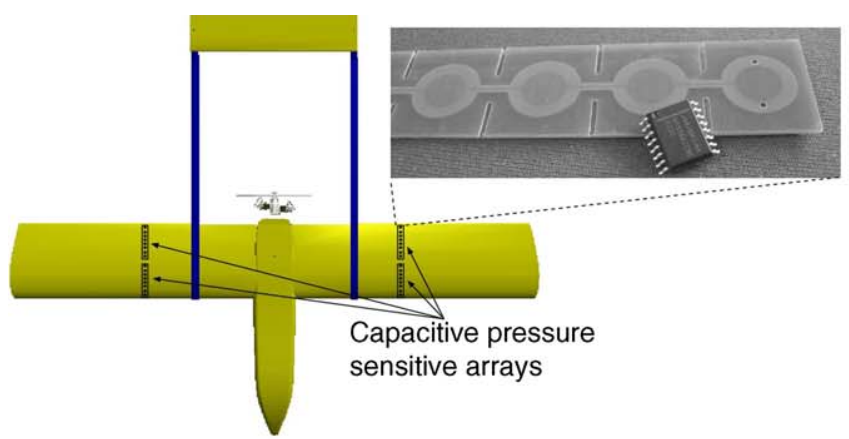

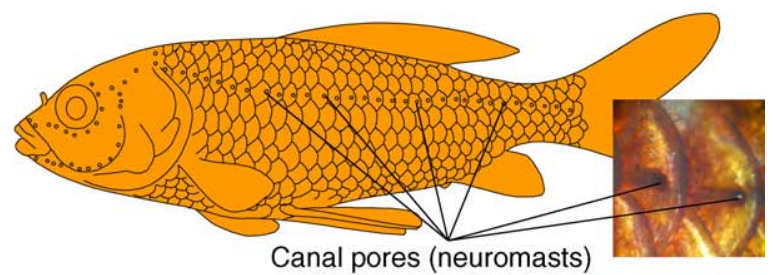

(B)

(A)

Fig. 1. Analogy between a fluid-dynamic sensing systems used in Nature and the proposed approach: (A) proposed instrumentation to be applied on the aerodynamic surfaces of an aircraft; (B) lateral line of neuromasts (nanoscale fluidic sensors) used by some fishes and amphibians to infer swimming attitude and other information.

straints are relaxed, since the existence of regions of the flight envelope where some sensors are perturbed can be treated as a transient failure of a minority of units.

For transducing, the proposal relies on conformable strips hosting capacitive pressure sensitive units, directly placed on the aircraft aerodynamic surfaces as shown in Fig. 1(A). In many senses, this approach can be considered more "natural" than conventional ones. In fact, Nature often uses large arrays of simple sensing elements in conjunction with sophisticated signal processing. Particularly, fishes and aquatic amphibians know about their attitude in water as a part of the signal processing practiced by the lateral system which involves a plurality of elementary pressure and shear sensors (neuromasts) aligned on their body, as shown in Fig. 1(B) [6]. ${ }^{1}$ Analogy with the proposed system is remarkable.

In this paper, the analysis is limited to probes located on airfoil sections but the generalization to three-dimensional bodies (and thus to side-slip detection) is possible. It is worth mentioning that attempts at exploiting distributed sensors surveying local pressures have already proven successful to the inference of high-level aeronautic data [7].

The design of the sensor system described in this paper follows many parallel tracks, reflected by the paper organization as follows:

- Section 2 summarizes the development of a suitable sensing technology, following the approach presented in [8] which focuses on the application of Printed Circuit Board (PCB) technologies to the implementation of pressure sensors.

- Section 3 describes the mathematical methods used in the system and illustrates the development of suitable algorithms and data structures, expanding the approach preliminarily presented in the conference paper [9]. Specifically, an error propagation model leading from uncertainties on pressure readings to final uncertainties on flight parameters is here fully developed and techniques for fault detection and fault tolerance are introduced.

\footnotetext{
${ }^{1}$ In nature, signal processing is so evolved that the lateral system can provide information related to obstacle avoiding or prey detection.
}

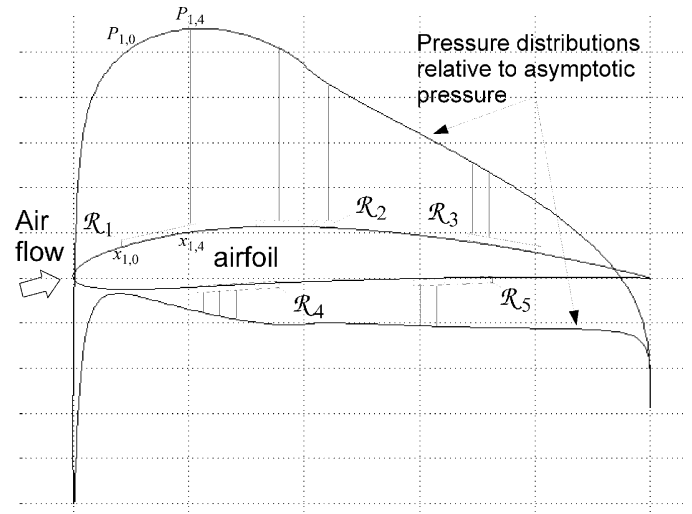

Fig. 2. Sample placement of "region of interest" and spots where information about pressure gradients over the airfoil is returned.

- Section 4 describes validation methods. These include simulation and wind tunnel experiments. For convenience, the latter can employ conventional instrumentation (as reported in the conference paper [10]). However, here we also present the results of the first wind tunnel tests based on real sensor prototypes.

\section{Sensing technologies}

As it will get clear in Section 3, the proposed measurement system requires sensors capable of delivering partial views of the pressure gradients applied by the airflow over the aircraft surfaces. It is assumed that such views are provided in the form of vectors each of which represents a spatial discretization of the pressure gradient at some location. Fig. 2 helps to illustrate this assumption by showing an airfoil section where five sample "regions of interest" ( $\mathcal{R}_{1}$ to $\left.\mathcal{R}_{5}\right)$ are marked. For each region $\mathcal{R}_{j}$, specific spots $x_{j, 0}$ to $x_{j, n}$ exist, contributing to the spatial discretization. With this, the sensor in charge of region $\mathcal{R}_{j}$ is expected to return a vector ${ }^{2} \vec{P}_{j}=\left(P_{j, 1}, \ldots, P_{j, n-1}\right)^{\mathrm{T}}$ where entry $P_{j, i}$ represents the pressure difference between the spots $x_{j, i}$ and $x_{j, 0}$. The vectors $\vec{P}_{j}$ can then be concatenated into a single

\footnotetext{
${ }^{2}$ Here and in the following, the matrix transposition operator ${ }^{\mathrm{T}}$ is used to represent column vectors more compactly.
} 


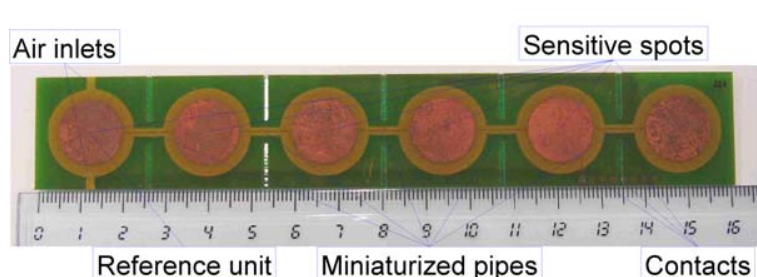

(A)

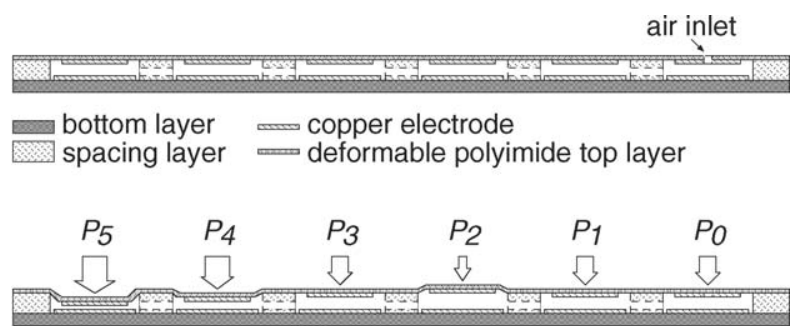

(B)

Fig. 3. Strip sensor array in PCB technology. Prototype photography (A); section and schematic operation (B). Sensor readout is capacitive.

vector of readings $\vec{R}$. Note that $\vec{R}$ does not represent a pressure gradient itself, as the pressure differences between the various locations $\mathcal{R}_{j}$ need not to be known.

From the above, it should be clear that the requirements over the sensor sub-system are particularly mild. First of all, sensors can be differential as one is interested in pressure gradients and not in absolute distributions. This can greatly simplify the sensor design, neglecting absolute pressures which can significantly vary with altitude and meteorological conditions. Secondly, the sensors in charge of the many regions can be independent of each other as no pressure difference needs to be evaluated between them.

Evidently, many technologies can be used for satisfying the above requirements. For instance, one could drill orifice arrays in the regions of interests and pneumatically link them to conventional multi-point pressure scanners [3,11]. Alternatively, one could think of sensors based on silicon micro-electromechanical systems (MEMS) and/or hybrid technologies [12]. In practice, however, one should make the sensor system as cheap, robust and easy to install as possible. To this aim, sensor strips based on PCB technologies are being prototyped and tested, as reported in [8], and as shown in Fig. 3. These are three-layer structures where the mid-layer is shaped to make the device hollow and organized in chambers. The chambers can communicate with each other and with a pressure inlet, so that the same reference pressure can be propagated within them. Their top is flexible, so that it can operate as a membrane, deforming according to the pressure difference between the inside and the outside. The chamber floor and ceiling are metal plated, so that such deformation can be read as capacitance variations. The structure can be made conformable to mildly curved surfaces, less than $1 \mathrm{~mm}$ thick (minimizing flow perturbations) and in length between $10 \mathrm{~cm}$ and over $50 \mathrm{~cm}$, depending on manufacturing facilities. Furthermore, good mechanical robustness can be achieved. The fact that each strip hosts many units is noteworthy, as it lowers manufacturing costs and eases installation, favoring the deployment of very redundant setups. Furthermore, it enables a strip to directly represent a "regions of interest" so that from the vector $\vec{C}_{j}$ of its capacitance readings one can immediately get the corresponding vector $\vec{P}_{j}$, once the characteristics of the elementary transducers is determined/assigned $[8,13]$. The resolution is expected to be about 5-10 $\mathrm{Pa}$ over a range spanning over $1 \mathrm{kPa}$ (7-8 bit of effective resolution), which is sufficient for air speed and angle of attack detection, as shown in the following sections. Note that the sensor reading can be performed by analog interface circuits carrying out capacity measurement and A/D conversion. The collection of data thus needs only electric means and can require as little as a couple of wires shared among all sensors if serial communication is adopted. The interested reader is invited to check [8] and [14] which offer an in-depth view of the sensing strips and interfacing.

\section{Theory of operation}

From the above, it should be clear that $m$ strip sensors with $n$ sensitive spots each ( $n-1$ pressure differences per sensor) represent $m$ regions of interest and deliver a compound readout vector

$\vec{R}=\left(\begin{array}{c}\Delta \vec{P}_{1} \\ \vdots \\ \Delta \vec{P}_{m}\end{array}\right)=\left(\Delta P_{1,1}, \ldots, \Delta P_{1, n-1}, \Delta P_{2,1}, \ldots\right.$,

$\left.\Delta P_{2, n-1}, \ldots, \Delta P_{m, n-1}\right)^{\mathrm{T}}$

The vector $\vec{R}$ thus holds $l=m(n-1)$ elements where $n$ can typically be any integer between two and a few tens. ${ }^{3}$

The signal processing aspects involve the definition of algorithms to properly consume this data and return high-level flight parameters including the free-stream air speed $\left(V_{\infty}\right)$ and the angle of attack $(\alpha)$. In general terms, one can think of a vector $\vec{\Phi}=\left(V_{\infty}, \alpha\right)^{\mathrm{T}}$ of quantities of interest with the provision of extending it to include the side-slip angle $\beta$ too. ${ }^{4}$ The algorithms should exploit the functional tie that must exist between $\vec{\Phi}$ and $\vec{R}$, and which can be captured in most general terms as

$\vec{R}=\boldsymbol{F}(\vec{\Phi}, \vec{E})$

where $\vec{E}$ is a vector of environmental parameters that might affect the tie (e.g. air density). In the following, dependence on $\vec{E}$ will be implicitly assumed without marking it, to keep the notation simpler.

\footnotetext{
${ }^{3}$ Alternatively, and with trivial modifications to the following mathematical methods, one can think of filling $\vec{R}$ directly with the capacitive readings of the strip sensors as $\vec{R}=\left(C_{1,1}, \ldots, C_{1, n-1}, C_{2,1}, \ldots, C_{2, n-1}, \ldots, C_{m, n-1}\right)^{\mathrm{T}}$.

${ }^{4}$ To this aim it is clearly not enough to fit horizontal airfoil surfaces with sensors, and one needs also to define "region of interests" on vertical planes. However, no structural modifications to the mathematical methodologies presented in this paper are strictly needed.
} 


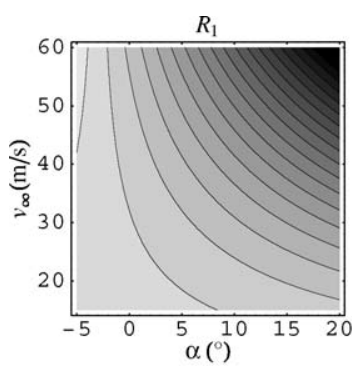

$R_{3}$

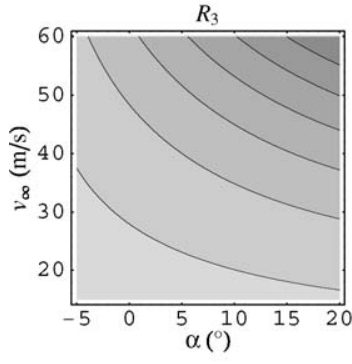

(A)
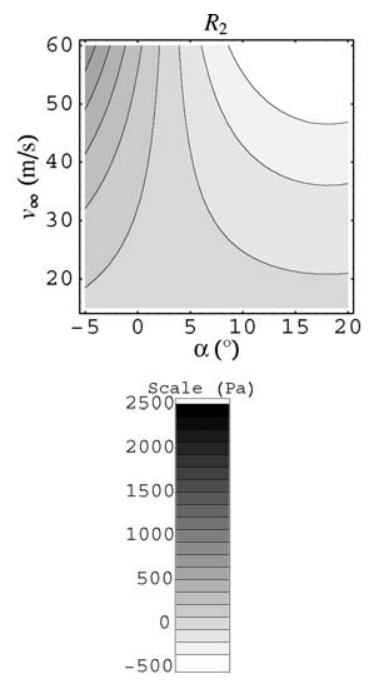

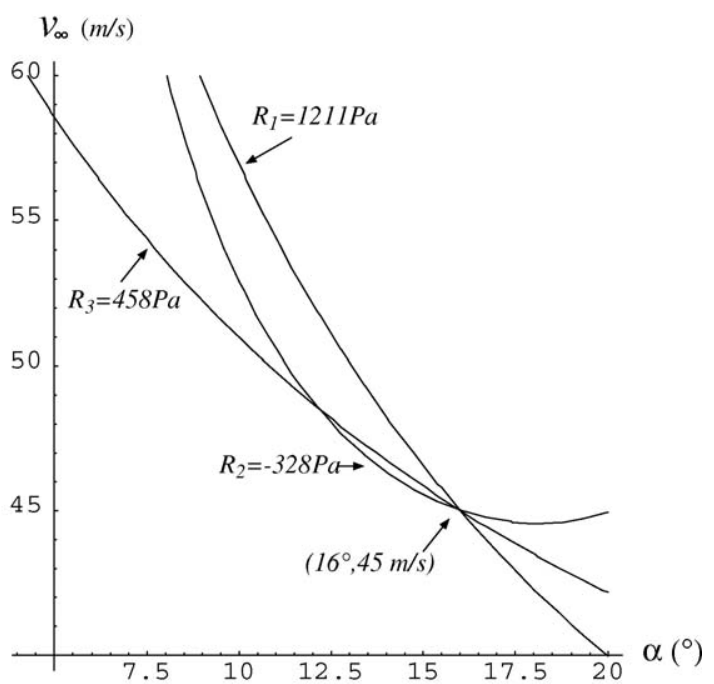

(B)

Fig. 4. (A) Contour plots of the various components of $\boldsymbol{F}$ for a test case where $l=3$. (B) Corresponding graphical determination of attack angle and air speed for a readout vector $\vec{R}$ whose components are those on the corresponding isoclines. Test conditions: data obtained by simulation for a normalized NACA 0012 airfoil at room pressure and temperature. Airfoil is equipped with three two-units sensor strips: first strip on top surface, with sensing spots at $x_{1,0}=0.2 \mathrm{~m}$ and $x_{1,1}=0.4 \mathrm{~m}$ from leading edge; second strip on bottom surface with $x_{2,0}=0.2 \mathrm{~m}$ and $x_{2,1}=0.4 \mathrm{~m}$; third strip on top surface with spots at $x_{3,0}=0.7 \mathrm{~m}$ and $x_{3,1}=0.9 \mathrm{~m}$.

Note that knowledge about $\boldsymbol{F}$ can be obtained by various means, e.g. exploiting analytical methods (by fluid-dynamic modeling), by simulation or measurements (sampling the space of definition of $\vec{\Phi}$ at significant points and applying interpolation) or by mixture of these techniques. Assuming that $\boldsymbol{F}$ is known, one should ideally use its inverse to compute $\vec{\Phi}$ once a reading vector $\vec{R}$ is delivered by the sensor system. The determination of $\boldsymbol{F}$ is thus crucial and can be regarded as a calibration phase. For the moment, assume that $\boldsymbol{F}$ is known and $\mathcal{C}^{1}$ in $\vec{\Phi}$. With this, the first issue to clear is to assess that $\boldsymbol{F}$ is invertible.

In intuitive terms the inversion problem consists in taking the various components of $\boldsymbol{F}$, as in Fig. 4(A), and in finding the intersection point of their isoclines corresponding to the components of the sensor readout vector $\vec{R}=\left(R_{1}, \ldots, R_{l}\right)^{\mathrm{T}}$ as in $F_{1}(\vec{\Phi})=R_{1}, \ldots, F_{l}(\vec{\Phi})=R_{l}$. This is shown in Fig. 4(B). From the example, it is evident that the invertibility of $\boldsymbol{F}$ cannot be generally assured. For instance, if in Fig. 4(B) one had considered only two isoclines (ignoring the output of a sensing unit), there would have been two intersection points and inversion ambiguity. For some specific sensor placements proving invertibility might be possible, but deriving invertibility conditions for arbitrary sensor placements and numbers would most likely be cumbersome. Fortunately, this is practically not needed as one can rely on the probability of $\boldsymbol{F}$ being not invertible to decrease extremely rapidly when the number of sensing units $l$ is increased. In fact the diversity among the components of $\boldsymbol{F}$ makes it extremely hard not to be one-to-one when $l$ is large. Hence, one can simply consider invertibility not be an issue as long as the proposed measurement system uses a sufficiently large number of sensing units, as it is actually meant to do.

Once invertibility is assessed, one needs to consider how to exploit this property. In fact, naive inversion approaches cannot be practiced due to uncertainties affecting the readout of $\vec{R}$.
Unavoidable measurement errors have the consequence that one cannot operate on $\vec{R}$ directly. Only "perturbed instances" $\vec{S}$ of $\vec{R}$ are available, built as

$$
\begin{aligned}
\vec{S}= & \left(\Delta P_{1,1}+\varepsilon_{1,1}, \ldots, \Delta P_{1, n-1}+\varepsilon_{1, n-1}, \Delta P_{2,1}+\varepsilon_{2,1}, \ldots,\right. \\
& \left.\Delta P_{2, n-1}+\varepsilon_{2, n-1}, \ldots, \Delta P_{m, n-1}+\varepsilon_{m, n-1}\right)^{\mathrm{T}}
\end{aligned}
$$

where the vector $\vec{\varepsilon}=\left(\varepsilon_{1,1}, \ldots, \varepsilon_{m, n-1}\right)^{\mathrm{T}}$ summarizes the reading errors. Keeping reference to the example in Fig. 4 , their effect is to make it virtually impossible to have a single point where all the isoclines of the $\boldsymbol{F}$ components intersect.

This fact can be seen from another perspective by considering that $\boldsymbol{F}$ maps points from $\mathbb{R}^{q}$ (where $q$ is the dimensionality of $\vec{\Phi}$, e.g. (2)) to a subset $\mathcal{D}$ of $\mathbb{R}^{l}$ which is an $l$-dimensional manifold. ${ }^{5}$ As an example, Fig. 5(A) shows a possible image set of an interval $\left(V_{\infty}, \alpha\right)^{\mathrm{T}}$ through $\boldsymbol{F}$ when $l=3$.

In this light, one sees that reading errors make it unlikely for $\vec{S}$ to fall on the image set $\mathcal{D}$ of $\boldsymbol{F}$, so that even knowing $\boldsymbol{F}^{-1}: \mathcal{D} \mapsto \mathbb{R}^{q}$ one could not employ it to compute $\vec{\Phi}$.

In conclusion, one is left with a classical estimation problem [15]. A very practical approach to its solution is maximum likelihood estimation (MLE). The approach requires knowing the probability density function (PDF) of $\vec{S}$ once $\vec{\Phi}$ is assumed to be deterministically set at a given value. Such $\operatorname{PDF} \rho(\vec{S} ; \vec{\Phi})$ is termed a likelihood function whenever it is viewed as a function of the unknown $\vec{\Phi}$ with $\vec{S}$ fixed. MLE is hence practiced by maximizing $\rho(\vec{S} ; \vec{\Phi})$ in $\vec{\Phi}$ when $\vec{S}$ is given. In the specific estimation problem being tackled, the first step is to make reasonable hypothesis about the statistical distribution of $\vec{\varepsilon}$, since this enters the definition of $\rho(\vec{S} ; \vec{\Phi})$. It seems reasonable to assume $\vec{\varepsilon}$ to be

${ }^{5}$ Excluding a pathological case where $l$ is 1 . 


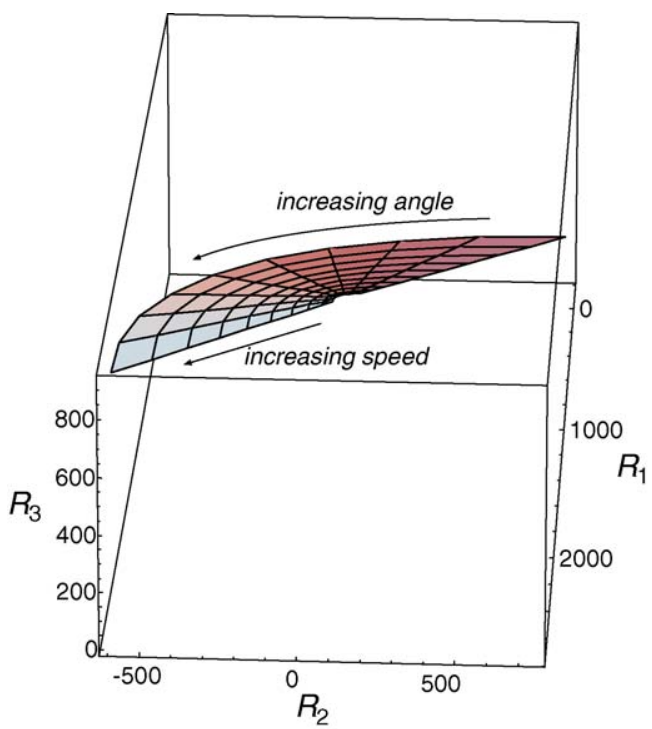

(A)

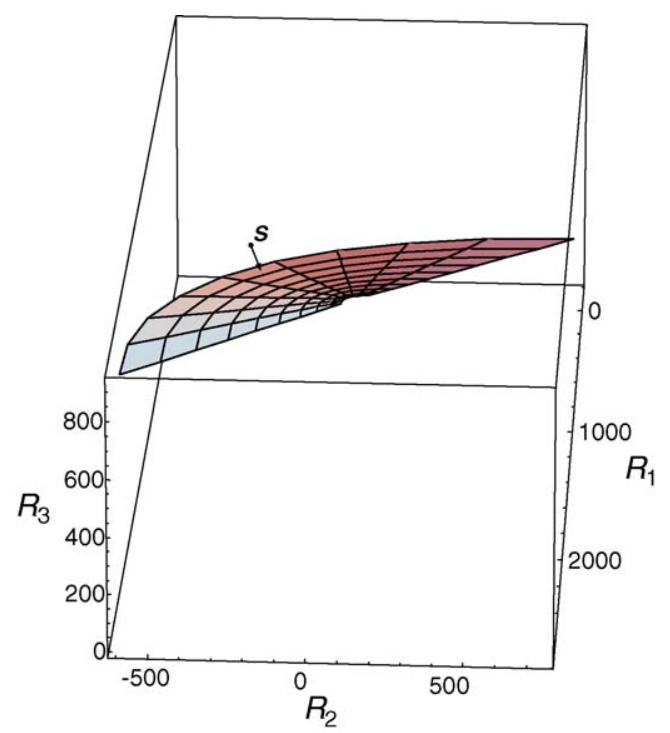

(B)

Fig. 5. (A) Image set of an interval in the $\vec{\Phi}=\left(V_{\infty}, \alpha\right)^{\mathrm{T}}$ plane through function $\boldsymbol{F}$. Interval is defined by $\alpha \in\left[-5^{\circ},+8^{\circ}\right]$ and $V_{\infty} \in[15,60] \mathrm{m} / \mathrm{s}$. (B) Projection operator used for the inversion of $\boldsymbol{F}$ when the reading vector is affected by measurement errors. Test conditions are the same as for the previous figure.

independent from $\vec{\Phi}$ (at least in a first order approximation). Furthermore, presuming that the various sensing units are identical and correctly built not to influence each other, it seems also reasonable to assume that the many $\varepsilon_{i, j}$ are independent identical distributed (IID) random variables, so that the first order statistics of $\vec{\varepsilon}$ can easily be factorized. Additionally, assuming that $\varepsilon_{i, j}$ are non-systematic, it seems reasonable to assume $\varepsilon_{i, j} \sim \mathcal{N}\left(0, \sigma_{\varepsilon}\right)$, i.e. that errors distribute normally with zero mean and a certain variance $\sigma_{\varepsilon}^{2}$. With all this:

$\rho(\vec{S} ; \vec{\Phi})=\frac{1}{\left(2 \pi \sigma_{\varepsilon}^{2}\right)^{1 / 2}} \exp \left[-\frac{1}{2 \sigma_{\varepsilon}^{2}} \sum_{i=1}^{l}\left(S_{i}-F_{i}(\vec{\Phi})\right)^{2}\right]$

where $S_{i}$ and $F_{i}$ are the components of $\vec{S}$ and $\boldsymbol{F}$, respectively.

Note that the sum in (4) is just the squared euclidean distance $\|\boldsymbol{F}(\vec{\Phi})-\vec{S}\|$. Hence, the maximization of (4) can be conveniently restated as

$$
\underset{\text { in } \vec{\Phi}}{\operatorname{minimize}}\|\boldsymbol{F}(\vec{\Phi})-\vec{S}\|
$$

The minimization can be performed numerically [16], for instance by gradient descent or Newton methods. However, if $\boldsymbol{F}$ is modeled by an interval-wise polynomial function, exact solutions may also be achievable. The MLE expression (5) lends to a further interpretation of the estimation mechanism. Since $\boldsymbol{F}^{-1}$ is not sufficient by itself for the inversion problem in presence of reading errors one needs a generalization $\boldsymbol{G}: \mathbb{R}^{l} \mapsto \mathbb{R}^{q}$ of $\boldsymbol{F}^{-1}$ in order to be able to deal with them. Since the proposed MLE framework - with the assumed error model - reduces to least square estimation by (5), the optimal solution is clearly represented by defining $\boldsymbol{G}$ as the combination of $\boldsymbol{F}^{-1}$ with an orthogonal projection operator $\boldsymbol{T}$ taking $\vec{S}$ onto $\mathcal{D}$ as shown in Fig. 5(B). In other terms $\boldsymbol{F}^{-1}$ gets applied to the point of $\mathcal{D}$ closest to the reading $\vec{S}$. While this interpretation is not convenient for the practical implementation of a computation algorithm, it can be useful for the prediction of estimation uncertainties, as shown in the following Section 3.3.

\subsection{System extension to fault detection and to fault tolerant operation}

After the optimization (5), the quantity object of minimization can be employed as a merit factor to qualify the estimation procedure. Under the assumption that the components of $\vec{\varepsilon}$ are IID and distributed according to $\mathcal{N}\left(0, \sigma_{\varepsilon}\right)$, for each given $\vec{\Phi}$, the quantity $\|\boldsymbol{F}(\vec{\Phi})-\vec{S}\|$ can be expected to be a random variable with a Chi-like distribution. Let us indicate by $\sigma_{\mathrm{CS}}^{(l)}(x)$ the probability density function of the normalized Chi-square distribution with $l$ degrees of freedom and define a quantity $d_{\max }$ so that

$\int_{0}^{d_{\max }^{2}} \frac{1}{\sigma_{\varepsilon}^{2}} \rho_{\mathrm{CS}}^{(l)}\left(\frac{x}{\sigma_{\varepsilon}^{2}}\right) \mathrm{d} x=\theta$

where $\theta$ is a quantity close to 1 (e.g. 99\%). With this, the probability that a measure vector $\vec{S}$ leads to a $\|\boldsymbol{F}(\vec{\Phi})-\vec{S}\|>d_{\max }$ should be less than $1-\theta$ (e.g. less than $1 \%)$. This consideration opens the way to a convenient fault detection mechanism for the proposed air data system. Whenever the quantity minimized in (5) exceeds a threshold ( $d_{\max }$ or presumably a larger quantity to have some clearance), one knows that $\vec{S}$ is degraded by anomalously large errors. The latter might be caused by sensor faults, data transmission errors, or transient errors due to flow perturbations or wake effects. ${ }^{6}$ In mission-critical systems the ability to derive a reliability index from measured data can be a fundamental feature. Note that this comes as a direct benefit of the exploitation of a redundant sensor set where incoherence among the sensor readings can trigger alerts. If the sensor redundancy

\footnotetext{
${ }^{6}$ The approach is also able to detect convergence problems in the minimization algorithm if numerical computation methods are used.
} 
is large enough, the fault detection mechanism can be easily extended to achieve fault tolerance. In fact, when $\|\boldsymbol{F}(\vec{\Phi})-\vec{S}\|$ is too large, one can progressively exclude some sensor readings from the estimation phase, until the minimization residue goes back to normality. At this point, the faulty part of the system is isolated.

\subsection{System calibration}

Having discussed the usage of function $\boldsymbol{F}$ for the estimation of $\vec{\Phi}$, it is worth considering the determination of $\boldsymbol{F}$ itself. Obviously, $\boldsymbol{F}$ depends on the particular shape of the aerodynamic surfaces on which the sensors are placed and on their particular locations. Note that the possibility of obtaining a closed-form $\boldsymbol{F}$ from physical models is generally hindered by the complications of fluid-dynamics. For instance, in [3] it was possible to rely on analytical models only thanks to the usage of particularly simple aerodynamic shapes. In this paper, $\boldsymbol{F}$ is approximated by using results from simulation and experimental data. Particularly, $\boldsymbol{F}$ is expressed by a $\underline{\boldsymbol{F}}(\vec{\Phi}, \vec{E}, \vec{K})$ where $\boldsymbol{F}$ represents a fixed functional structure and $\vec{K}$ is a vector of calibration parameters. In other terms a compact model is sought for the physical phenomena. The choice of the structure $\boldsymbol{F}$ is extremely important as it strongly affects the possibility of accurately expressing $\boldsymbol{F}$ with a minimal number of parameters and to calibrate it by a minimal set of experimental data. Furthermore, the form of $\boldsymbol{F}$ influences the possibility of efficiently computing the minimization (5). As already mentioned, the latter can normally be performed by numerical methods such as gradient descent of Newton algorithms, but for particular expressions of $\boldsymbol{F}$ (e.g. interval-wise polynomial) exact solutions might also be achievable. The latter may result particularly advantageous thanks to the possibility of pre-determining computation times, which favors the set up of real-time systems.

In this paper a preliminary approach has been pursued in order to get a proof of principle of the proposed techniques. This involves the definition of a limited region of $\vec{\Phi}$ points admissible in a realistic flight envelope (e.g. $\alpha \in\left[-8^{\circ}, 8^{\circ}\right]$ and $\left.V_{\infty} \in[20,45] \mathrm{m} / \mathrm{s}\right)$. In this region, significant sample points are identified by uniformly stepping $V_{\infty}$ and $\alpha$. At these points, pressure data is obtained either by simulation or by wind tunnel experiments. This data is then used to obtain an optimal $\vec{K}$ by fitting techniques, when $\boldsymbol{F}$ is set to have an intervalwise polynomial form (3rd order). Fluid-dynamic considerations can then be used to introduce dependencies on environmental parameters such as temperature, barometric pressure, etc. Clearly, this approximation method is almost uninformed of the underlying fluid-dynamic phenomena, and as such open to many optimizations. In the future, more sophisticated approximation techniques should be introduced to reduce the sets of experimental data needed for calibration. With the current approach, sample algorithm implementations have shown that a commodity computer is capable of delivering a solution to the estimation problem in about $0.1 \mathrm{~s}$ when the readout space is 10-dimensional $(l=10)$. Such result is obtained by applying an unspecialized minimization algorithm in an interpreted software environment, so significant computation time reductions should be at hand.

\subsection{Estimation of accuracy bounds}

In order to proficiently exploit the techniques described in the previous section, one should be able to forecast how the sensors reading errors propagate onto the final $\vec{\Phi}$ values. It is intuitive that, due to the inherent non-linearity of the computations, errors must propagate differently in different areas of the flight envelope.

A common index used in estimation theory is the Cramer-Rao lower bound (CRLB) [15]. Although it is a bound and not an exact variance evaluation, its usage can offer significant insight. Particularly, it alerts to the physical impossibility of finding unbiased estimators whose variance is less than its amount.

In general terms the CRLB states that:

$\operatorname{var}\left(\hat{\theta}_{i}\right) \geq\left[\boldsymbol{I}^{-1}(\vec{\theta})\right]_{i, i}$

where $\vec{\theta}$ is the vector of the parameters to estimate, $\hat{\theta}_{i}$ is an estimated parameter and $\boldsymbol{I}$ is the Fisher information matrix defined as

$[\boldsymbol{I}(\vec{\theta})]_{i, j}=-E\left[\frac{\partial^{2} \ln \rho(\vec{x} ; \vec{\theta})}{\partial \theta_{i} \partial \theta_{j}}\right]$

where $\vec{x}$ is the vector of observations on which the estimation is based. The specialization of the CRLB to the estimation problem described above has a particularly compact form thanks to the hypotheses made on measurement errors: considering that the covariance matrix of the observations is diagonal with entries $\sigma_{\varepsilon}^{2}$, that $\vec{\theta}$ is $\vec{\Phi}$ and that $\vec{x}$ is $\vec{S}$, one has:

$\boldsymbol{I}(\vec{\Phi})=\frac{1}{\sigma_{\varepsilon}^{2}} \boldsymbol{X}$

where $\boldsymbol{X}=(\mathbf{J}[\boldsymbol{F}])^{\mathrm{T}} \mathbf{J}[\boldsymbol{F}]$ and $\mathbf{J}[\boldsymbol{F}]$ is the Jacobian matrix of $\boldsymbol{F}$. From this, when $\vec{\Phi}=\left(V_{\infty}, \alpha\right)^{\mathrm{T}}$

$$
\begin{aligned}
& \sigma_{V_{\infty}} \geq \sigma_{\varepsilon} \sqrt{\left[\left((\boldsymbol{J}[\boldsymbol{F}])^{\mathrm{T}} \mathbf{J}[\boldsymbol{F}]\right)^{-1}\right]_{1,1}} ; \\
& \sigma_{\alpha} \geq \sigma_{\varepsilon} \sqrt{\left[\left((\mathbf{J}[\boldsymbol{F}])^{\mathrm{T}} \mathbf{J}[\boldsymbol{F}]\right)^{-1}\right]_{2,2}}
\end{aligned}
$$

where $\sigma_{V_{\infty}}$ and $\sigma_{\alpha}$ are the standard deviations to expect on $V_{\infty}$ and $\alpha$, respectively.

It is also worth considering that in the particular application, the CRLB can be attained in an approximate sense. In other terms it can be used to forecast the effective accuracy that can be obtained from the proposed air data system, given the sensors characteristics (and thus $\sigma_{\varepsilon}$ ), number and location. Once accuracy specifications are provided for the whole system this can be precious to chose the sensors to adopt and to optimize their placement. The possibility to use (10) as an excellent approximation of the effective performance of the MLE for the proposed application derives from $\boldsymbol{F}$ being locally not too far from linear (almost quadratic in $V_{\infty}$ and smooth in $\alpha$ ).

In geometrical terms, this can be seen by considering the framework of Fig. 5(B), where the estimation of $\vec{\Phi}$ is obtained as $\overrightarrow{\boldsymbol{F}}^{-1}(\overrightarrow{\boldsymbol{T}}(\vec{S}))=\boldsymbol{G}(\vec{S})$. If $\boldsymbol{F}$ is not too far from linear, in the neighborhood of any given $\vec{\Phi}$ it can be approximated by its 
linearization $\boldsymbol{F}_{\text {lin }}$, expressed by the Jacobian $\mathbf{J}[\boldsymbol{F}]$ at $\vec{\Phi}$. Note that the image set of $\boldsymbol{F}_{\text {lin }}$ is not $\mathcal{D}$, rather the (hyper)plane $\mathcal{D}_{\text {lin }}$ tangent to $\mathcal{D}$ at $\vec{R}=\boldsymbol{F}(\vec{\Phi})$. With this, one can approximate $\boldsymbol{G}(\vec{S})$ as $\boldsymbol{G}_{\text {lin }}(\vec{S})=\boldsymbol{F}_{\text {lin }}^{-1}\left(\boldsymbol{T}_{\text {lin }}(\vec{S})\right)$ where $\boldsymbol{T}_{\text {lin }}$ is a projection operator taking $\vec{S}$ onto $\mathcal{D}_{\text {lin. }}$. Since the columns of $\mathbf{J}[\boldsymbol{F}]$ define a basis for $\mathcal{D}_{\text {lin }}$, a variation of $\vec{S}, \Delta \vec{S}$, gets converted by $\boldsymbol{G}_{\text {lin }}$ into a variation of $\vec{\Phi}$ that can be expressed as $\Delta \vec{\Phi}=\left(\mathbf{J}[\boldsymbol{F}]^{\mathrm{T}} \mathbf{J}[\boldsymbol{F}]\right)^{-1} \mathbf{J}[\boldsymbol{F}]^{\mathrm{T}} \Delta \vec{S}$. With this formula, one can compute the covariance matrix for $\Delta \vec{\Phi}$, assuming a diagonal covariance matrix $\sigma_{\varepsilon}^{2} \mathbf{1}_{l}$ for $\Delta \vec{S}$ (where $\mathbf{1}_{l}$ is the $l \times l$ identity matrix). The covariance expression is

$$
\begin{aligned}
E & {\left[\Delta \vec{\Phi} \Delta \vec{\Phi}^{\mathrm{T}}\right] } \\
& =E\left[\left(\mathbf{J}[\boldsymbol{F}]^{\mathrm{T}} \mathbf{J}[\boldsymbol{F}]\right)^{-1} \mathbf{J}[\boldsymbol{F}]^{\mathrm{T}} \Delta \vec{S} \Delta \vec{S}^{\mathrm{T}} \mathbf{J}[\boldsymbol{F}]\left(\mathbf{J}[\boldsymbol{F}]^{\mathrm{T}} \mathbf{J}[\boldsymbol{F}]\right)^{-1}\right] \\
& =\left(\mathbf{J}[\boldsymbol{F}]^{\mathrm{T}} \mathbf{J}[\boldsymbol{F}]\right)^{-1} \mathbf{J}[\boldsymbol{F}]^{\mathrm{T}} \sigma_{\varepsilon}^{2} \mathbf{1}_{l} \mathbf{J}[\boldsymbol{F}]\left(\mathbf{J}[\boldsymbol{F}]^{\mathrm{T}} \mathbf{J}[\boldsymbol{F}]\right)^{-1} \\
& =\sigma_{\varepsilon}^{2}\left(\mathbf{J}[\boldsymbol{F}]^{\mathrm{T}} \mathbf{J}[\boldsymbol{F}]\right)^{-1}
\end{aligned}
$$

i.e. it is equivalent to (10) when equalities are substituted for the inequalities in (10) and one is only interested in the variance (diagonal) terms of (11).

Once the sensor accuracy is known and expressed as a standard deviation, formulas like (10) and (11) enable plots such as those of Fig. 6 to be drawn. Here, the pressure sensor accuracy is related to the global accuracy that can be obtained in the measurement of $\vec{\Phi}$, once the sensor placement is assigned. Obviously, the global accuracy that can be obtained depends on the specific point of the flight envelope where one operates. Notably, the accuracy is better at low angles of attack and at high speeds. This seems to be a major regularity, independent of the specific sensor placement being adopted.

As a final remark, note that the possibility of estimating the accuracy of the approach by a closed form expressions such as (10) and (11) can be fundamental in the practical design of an air data system. Particularly, expressions such as (10) and (11) enable the coding of optimization procedures where the placement of the sensors over the aerodynamic surfaces of an aircraft is incrementally adjusted until the best possible accuracy in the estimation of $\vec{\Phi}$ is obtained.

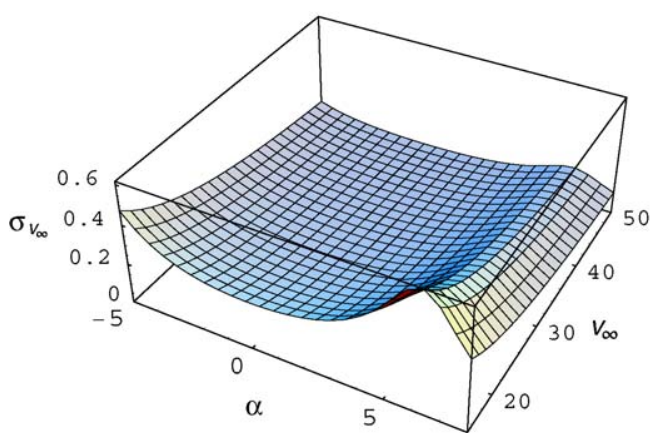

(A)

\section{Experimental results}

The methodologies described in the previous section have been validated by three independent means, involving both numerical simulation and experiments practiced in a wind tunnel. The usage of three different validation tracks can be very advantageous since different experiment types permit different level of control of environmental information and are characterized by different pieces of return information. For instance, wind tunnel experiments offer realistic operating conditions, while simulation-based techniques allow strict control of second order aerodynamic effect and exclude that equipment set up problems can compromise the interpretation of data. Furthermore, experimental tracks that do not require the strip pressure sensors to be physically available make it possible to start experiments before prototypes sensors are available, enabling the concurrent development of the software and hardware parts of the proposed measurement system.

\subsection{Simulation tests}

By simulation one gets "ideal" input data to which numbers obtained by wind tunnel experiments can then be confronted for interpretation. Furthermore, simulation allows one to check the hypothesis that the CRLB can be used to forecast flight parameters uncertainties, without interference from second order aerodynamic phenomena. Finally, simulation provides data useful to drive and optimize the sensor design.

The simulation tests herein reported consisted in the extensive application of Monte Carlo methods to accumulate sufficient information to derive the statistics of estimation errors. Simulation tests were practiced with the aid of the fluid-dynamic code Xfoil [17,18], linked to a Computer Algebra System by some ad hoc glue software. Particularly, the simulations procedure comprises the following steps:

1. A simulation setup is assigned, including an airfoil description, a sensor characterization and information about sensor placement over the airfoil.

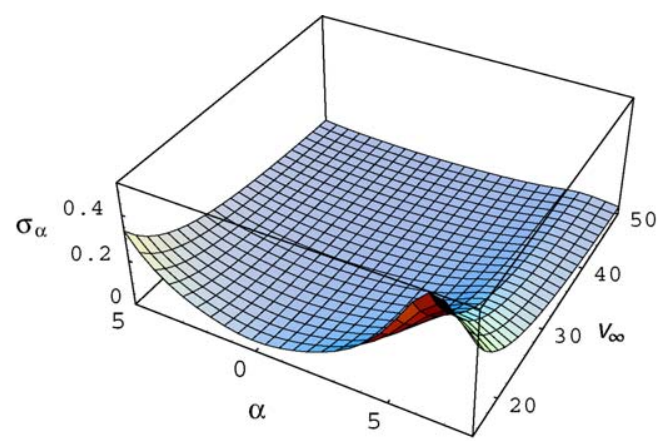

(B)

Fig. 6. Sample standard deviation surfaces obtained from Eqs. (10) and (11). (A) Standard deviation on $V_{\infty}$; (B): standard deviation on $\alpha$. Test conditions: data obtained by simulation for a NACA0012 airfoil $(50 \mathrm{~cm}$ chord) equipped with two symmetrically placed strip sensors with nine sensitive spots each: first strip is on top surface of the airfoil, second strip on bottom; sensitive spots positions are at $x \cdot, j=(5+2.5 \cdot j) \mathrm{cm}$ from leading edge. The sensor resolution is assumed to be eight bits $\left(\sigma_{\varepsilon} \approx 2 \mathrm{~Pa}\right.$ over a $1500 \mathrm{~Pa}$ range). The plots show the possibility of measuring the free-stream speed at an accuracy within $\pm 2 \mathrm{~m} / \mathrm{s}$ and the attack angle at an accuracy within $\pm 1^{\circ}$ for this particular sensor setup. 


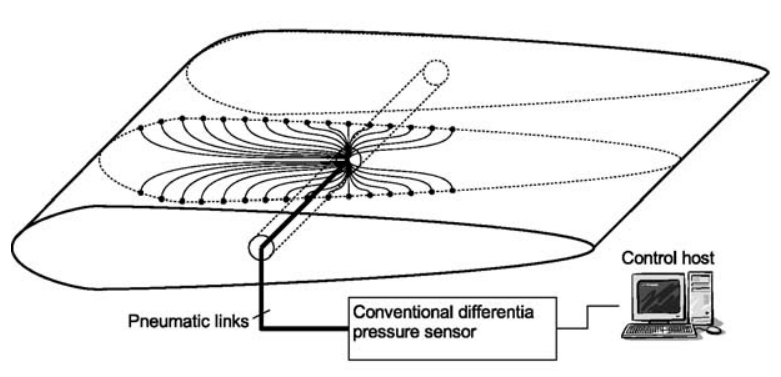

\begin{tabular}{|c|c|c|c|}
\hline \multicolumn{4}{|c|}{ Orifice placement from LE } \\
\hline$x_{\cdot, 0}$ & $x_{\cdot, 1}$ & $x_{\cdot, 2}$ & $x_{\cdot 3}$ \\
$32 \mathrm{~mm}$ & $45 \mathrm{~mm}$ & $58 \mathrm{~mm}$ & $71 \mathrm{~mm}$ \\
\hline$x_{\cdot, 4}$ & $x_{\cdot, 5}$ & $\mathrm{x}_{, 6}$ & $x_{\cdot, 7}$ \\
$84 \mathrm{~mm}$ & $97 \mathrm{~mm}$ & $110 \mathrm{~mm}$ & $123 \mathrm{~mm}$ \\
\hline$x_{\cdot, 8}$ & $x_{\cdot, 9}$ & $x_{\cdot, 10}$ & $x_{\cdot, 11}$ \\
$136 \mathrm{~mm}$ & $149 \mathrm{~mm}$ & $162 \mathrm{~mm}$ & $175 \mathrm{~mm}$ \\
\hline$x_{, 12}$ & $x_{\cdot, 13}$ & $x_{,}, 14$ & $x_{\cdot, 15}$ \\
$188 \mathrm{~mm}$ & $201 \mathrm{~mm}$ & $214 \mathrm{~mm}$ & $227 \mathrm{~mm}$ \\
\hline
\end{tabular}

Fig. 7. Model airfoil equipped with orifice arrays for the proposed experiments.

2. Xfoil is first run to create calibration data and to generate an approximating function $\overrightarrow{\boldsymbol{F}}(\vec{\Phi}, \vec{E}, \vec{K})$ as described in Section 3.

3. A cycle begins, including:

- the generation of a random nominal $\vec{\Phi}$;

- the usage of Xfoil to compute an ideal sensor reading vector $\vec{R}$ for such $\vec{\Phi}$

- the random perturbation of $\vec{R}$ to simulate measurement errors and to obtain a vector $\vec{S}$;

- the estimation of a vector $\overrightarrow{\hat{\Phi}}$ from $\vec{S}$;

- the recording of $\vec{\Phi}$ and the difference between the real $\vec{\Phi}$ and the estimated $\overrightarrow{\hat{\Phi}}$

4. The cycle is repeated many times $(>20,000)$.

5. At the end of the cycle standard deviations are computed for the estimation errors.

The above procedure confirmed the correctness of using the CRLB to forecast estimation uncertainties. Data obtained by simulation was coherent with analytically derived data such as that in Fig. 6.

\subsection{Tests with conventional instrumentation}

Tests with conventional instrumentation consist in creating a realistic wind tunnel setup. The tests employ an airfoil model. However, in this experimental track the airfoil is not yet equipped with capacitive strip sensors. Conversely, orifice arrays are drilled on its surfaces and linked to a conventional multi-point pressure scanner. The orifice arrays are then used to emulate the readings of the capacitive strip sensors.

The advantages of this "intermediate" experimental approach are two-fold. First of all, one can check the flight parameter inference algorithm relaying on well-acceded and documented instrumentation. Secondly, one can build data to be used as a benchmark for the further experiments using the real capacitive sensors. In this way, potential issues limited to the capacitive strip sensors can be easily isolated.

The experiments herein reported employed a NACA0015 ( $310 \mathrm{~mm}$ chord) airfoil model equipped with two symmetrically placed arrays of orifices emulating the readings of two 16-units PCB strips, as shown in Fig. 7. The right side of the figure also reports the position of the individual orifices from the airfoil leading edge.
The experiment consisted in a training phase and in a validation phase. In the training phase, data was collected either from simulation or from wind tunnel instrumentation sampling the $\left(\alpha, V_{\infty}\right)$ region of interest at regular points $\left(1^{\circ}\right.$ and $5 \mathrm{~m} / \mathrm{s}$ steps $)$. Training data was used to extract two calibration sets $\vec{K}_{\mathrm{s}}$ and $\vec{K}_{\mathrm{e}}$ for $\underline{\boldsymbol{F}}(\vec{\Phi}, \vec{E}, \vec{K})$, referring to simulation data and experimental data, respectively. Then, in the validation phase, the profile was placed in the wind tunnel at unknown angle and air speed. A reading $\vec{S}$ was made from the pressure probes and used to infer $\alpha$ and $V_{\infty}$ as described in Section 3. Measurement of $\alpha$ and $V_{\infty}$, were also taken by the wind tunnel scales and compared. It is important to remark that calibration and validation were always practiced on different data sets.

Fig. 8 helps better perceiving the discrepancy between measurements made by estimation and by the wind tunnel scales, for the case where the calibration vector $\vec{K}_{\mathrm{e}}$ is used. The case with $\vec{K}_{\mathrm{S}}$ is substantially similar, with only slightly larger errors. The $x$-axis reports $\alpha$, the $y$-axis reports $V_{\infty}$, and the $z$-axis (vertical) reports the magnitude of the measurement errors.

Note the generally good agreement, with errors smaller than $1^{\circ}$ ( $\sim 6 \%$ of range) and $1 \mathrm{~m} / \mathrm{s}(\sim 2.5 \%$ of max tested speed). Also note that although the amount of data is insufficient for statistical considerations, the plots appear to show slightly larger discrepancies at higher speeds. This suggests that aerodynamic effects ignored by simulation may play a significant role. This is interesting, particularly when confronted with data based on error propagation analysis, such as that presented in Section 3.3 and Fig. 6, where errors were larger at lower speeds when the errors introduced by the sensors had larger relative importance. ${ }^{7}$

\subsection{Tests with strip sensors}

The last experimental track consists in tests based on real strip sensors prototypes in a wind tunnel setup. Hence, all the components of the proposed measurement system are finally

\footnotetext{
${ }^{7}$ Actually, the regular structure of errors in $\alpha$ deserves some observations, since it suggests that errors might be partially imputable to the experimental setup rather than the proposed measurement approach itself. In fact, the error propagation models devised in Section 3.3 indicates that errors on $\alpha$ and $V_{\infty}$ should have a random structure, where the patterns in Fig. 8 do not clearly fit. A possible explanation might be to ascribe part of the errors to the mechanical clearance of the system used to set the airfoil angular position in the wind tunnel. Modifications of the experimental setup shall help clarify this point.
} 


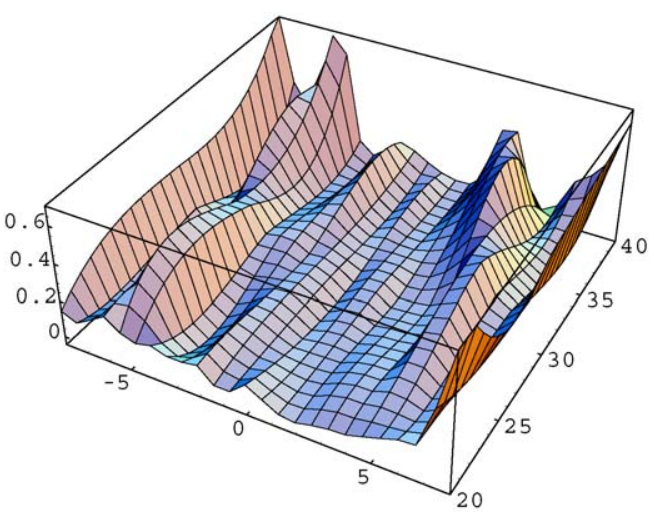

(A)

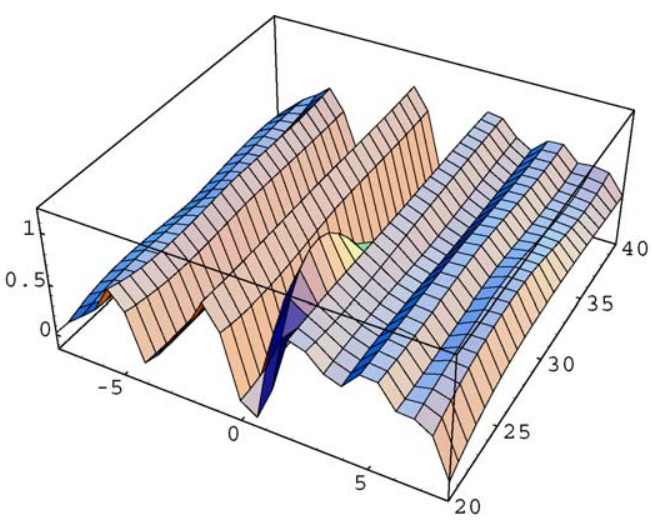

(B)

Fig. 8. Plots of discrepancy among data inferred by estimation and data obtained by conventional instruments. (A) Errors on $V_{\infty}$, in $\mathrm{m} / \mathrm{s}$; (B) errors on $\alpha$ in degrees.

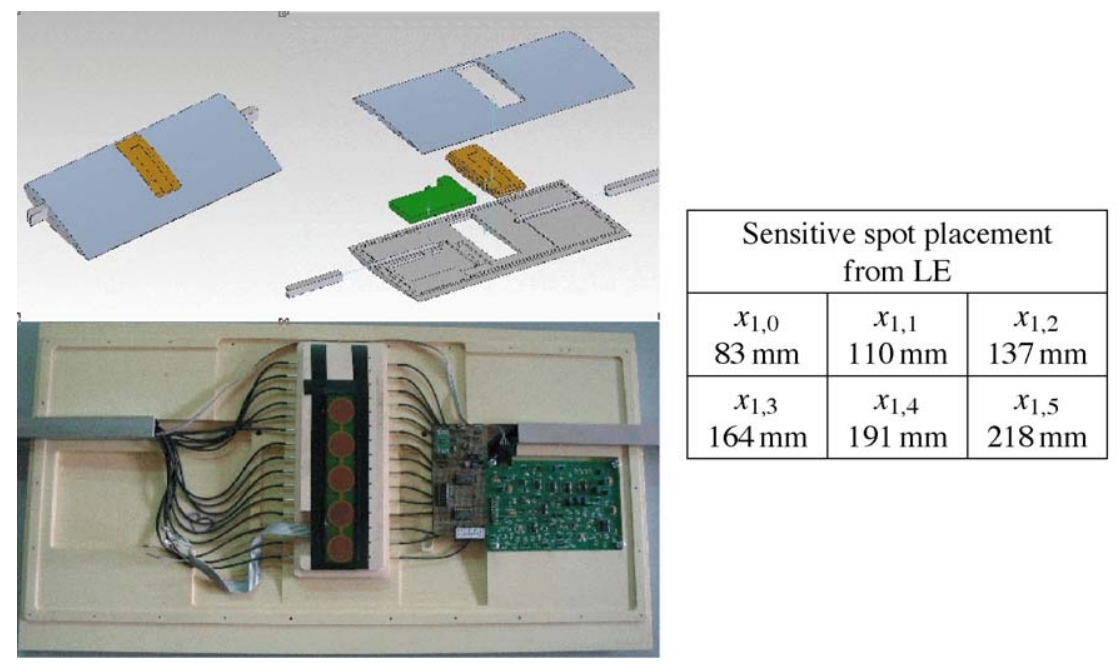

Fig. 9. Model airfoil equipped with a prototype strip sensor for the proposed experiments.

included. In this tests, the experimental setup is similar to the above, but a PCB strip sensor is glued on the skin of the model airfoil to be placed in the wind tunnel.

As above, the tests exploited a NACA 0015 airfoil $(310 \mathrm{~mm}$ chord). A single prototype PCB sensor was actually employed, being glued on the upper airfoil surface as illustrated in Fig. 9. The right side of the figure also report the exact placement of the sensitive spots.

The experiment followed the same guidelines of the previous section, with a notable exception: function $\underline{F}(\vec{\Phi}, \vec{E}, \vec{K})$ was taken to model a link between $\left(V_{\infty}, \alpha\right)$ and the sensor capacitance readings, not between $\left(V_{\infty}, \alpha\right)$ and pressure readings. ${ }^{8}$ In other terms $\overrightarrow{\boldsymbol{F}}$ was asked to incorporate the non-linear characteristics of the sensor, as pictured in Fig. 10. This could happen most naturally thanks to the extensive parametrization of $\overrightarrow{\boldsymbol{F}}$.

In operation the capacitance value for the sensing elements at rest were approximately $3.4 \mathrm{pF}$ (slightly varying among the many sensitive spots). Capacitance readings were made at $V_{\infty}$,

\footnotetext{
8 See also footnote 3
}

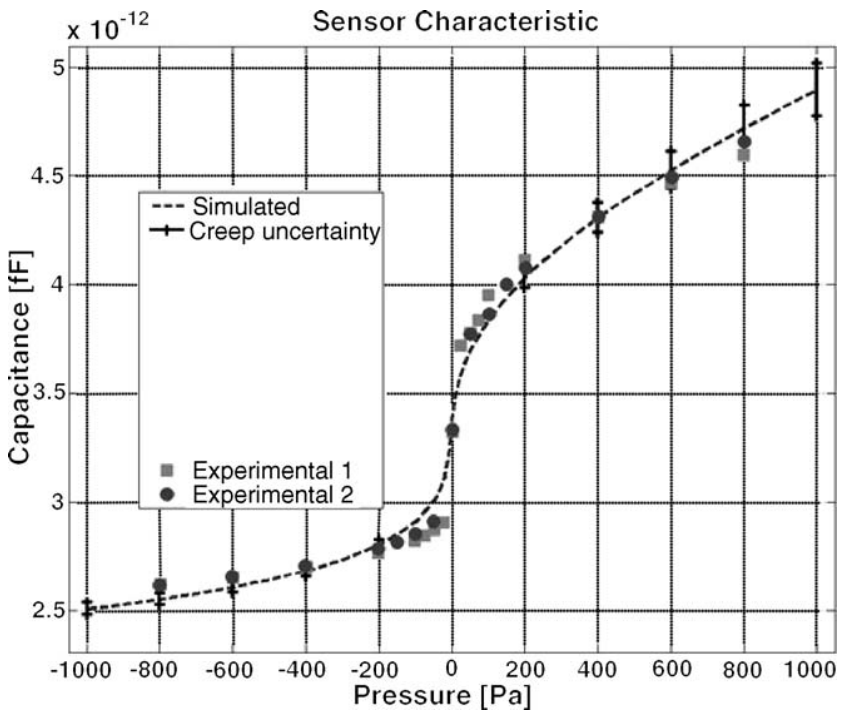

Fig. 10. Non-linear pressure to capacitance relationship of the prototype strip sensor used for the experiments. 
Table 1

Experimental verification of the correct operation of the proposed measurement system

\begin{tabular}{|c|c|c|c|c|c|}
\hline \multicolumn{2}{|l|}{ Setup } & \multicolumn{2}{|c|}{ Estimation } & \multicolumn{2}{|l|}{ Error } \\
\hline$V_{\infty}(\mathrm{m} / \mathrm{s})$ & $\alpha\left(^{\circ}\right)$ & $V_{\infty}(\mathrm{m} / \mathrm{s})$ & $\alpha\left(^{\circ}\right)$ & $\left|\Delta V_{\infty}\right|(\mathrm{m} / \mathrm{s})$ & $|\Delta \alpha|\left(^{\circ}\right)$ \\
\hline 30.15 & -4 & 30.08 & -4.04 & 0.07 & 0.04 \\
\hline 40.06 & -4 & 39.42 & -4.04 & 0.63 & 0.04 \\
\hline 29.91 & 0 & 29.14 & +0.30 & 0.78 & 0.30 \\
\hline 39.90 & 0 & 39.20 & +0.14 & 0.70 & 0.14 \\
\hline 30.10 & +4 & 30.80 & +3.17 & 0.70 & 0.82 \\
\hline 40.08 & +4 & 35.94 & +5.80 & 4.13 & 1.80 \\
\hline 29.91 & +8 & 30.04 & +7.97 & 0.13 & 0.03 \\
\hline 40.03 & +8 & 39.73 & +8.15 & 0.30 & 0.15 \\
\hline 0.09 & - & 0.45 & - & 0.37 & - \\
\hline
\end{tabular}

In tests, the set of measurements used for calibration was always disjoint from the measurements used in validation.

equal to $0,25,30,35,40 \mathrm{~m} / \mathrm{s}$ and $\alpha$ equal to $-8^{\circ},-4^{\circ}, 0^{\circ}, 4^{\circ}$, $8^{\circ}$. These test conditions made the sensor capacitance vary in a $[-500,+900] \mathrm{fF}$ range around their quiescent value. Capacitance measurements showed a repeatability of approximately $\pm 9 \mathrm{fF}$ ( $\pm 0.7 \%$ of the available range).

Table 1 shows the ability of the calibrated system to correctly infer $V_{\infty}$, and $\alpha$ at various speeds and angles of attack, resulting consistent with the theory presented so far. Errors are clearly larger than those presented in the previous section. This last track of experiments provided valuable results. Namely:

- Substantial coherence of the experimental data with data provided by theory and by other experimental tracks proved the generally correct operation of the capacitive sensors and the possibility of effectively using on-skin capacitive sensing for the measurement of high-level flight parameters.

- The possibility of seamlessly incorporating the non-linearities of the sensor model in the estimation procedure was shown.

- The possibility of reliably inferring high-level flight parameters even with the monitoring of a single airfoil surface was proved.

The larger estimation errors, obtained in this experimental track with regard to the previous one can be ascribed to some concurrent causes. First of all, it is worth noticing that the monitoring of a single airfoil surface reduces the diversity among the various sensor reading. From the CRLB-based error propagation analysis it is known that this negatively affects the final accuracy. Secondly, in this experiment the length of the readout vector $l$ was 5 , while in the previous track of experiments $l$ was 30 . From the CRLB-based error propagation analysis it could also be foreseen that a reduction in the input redundancy could negatively affect accuracy. Finally, the experiments revealed a second order phenomena in the sensors themselves. This is a long-term dynamical phenomena known as creep [8] that derives from the sensor materials, construction and sizing. In the future, results shall be improved also by contrasting this phenomena or accounting for it in the models.

\section{Conclusions and future work}

A novel measurement approach for air data has been presented, enabling the determination of free-stream air speed and flight attitude angles from measurement taken by a redundant set of identical pressure sensors directly applied on the skin of the aircraft. In the proposed methodology the aircraft itself is a part of the measurement system.

The theory of operation has been developed with the aid of estimation theory and accuracy bounds have been determined for the proposed approach. Experiments have been practiced in a wind tunnel to confirm the correctness of the theory. While the results obtained so far are satisfactory, research is still in progress, specifically dealing with the following goals:

1. Enhancement of the experimental equipment, to get a better perception of the achievable accuracy.

2. Enhancement of the experimental equipment, allowing airfoils to be equipped with many sensors at the same time, to test fault detection/fault tolerance schemes.

3. Extension of the methodology to side-slip detection.

4. Algorithm refinement and porting to an embeddable computing system.

5. Coding of procedures to help finding optimal sensor placement.

\section{References}

[1] M.A. Menzies, Integrated air data sensors, Aeronaut. J. 105 (2001) 223-229.

[2] F.J. Centolanzi, Characteristics of a $40^{\circ}$ cone for measuring Mach number, total pressure and flow angles at supersonic speeds, NACA, Technical Note 3967, 1957.

[3] S.A. Whitmore, Development of a pneumatic high-angle-of-attack flush air data sensing (HI-FADS) system, NASA, Memorandum 104241, November 1991.

[4] J. Choisnet, L. Collot, N. Hanson, Method for determining aerodynamic parameters and method for detecting failure of a probe used for determining aerodynamic parameters, US Patent Application 2004/0011124 A1, January 2004.

[5] S. Callegari, M. Zagnoni, A. Golfarelli, A. Talamelli, Apparecchiatura e metodo per la determinazione di parametri caratterizzanti il moto relativo di un corpo rispetto ad un fluido, University of Bologna, Italian Patent application for industrial invention BO2004A000647, October 2004.

[6] N.A.M. Schellart, R.J. Wubbels, The auditory and mechanosensory lateral line system, in: The Physiology of Fishes, 2nd ed., CRC Press, 1998.

[7] M. Holland, L. Eccles, L. Paradis, A pressure belt for airborne pressure survey, in: Proceedings of Sicon'01 (Sensors for Industry Conference), 2001, pp. 156-161.

[8] M. Zagnoni, A. Golfarelli, S. Callegari, A. Talamelli, V. Bonora, E. Sangiorgi, M. Tartagni, A non-invasive capacitive sensor strip for aerodynamic pressure measurement, Sens. Actuators A 123/124 (2005) 240-248.

[9] S. Callegari, A. Talamelli, M. Zagnoni, A. Golfarelli, V. Rossi, M. Tartagni, E. Sangiorgi, Aircraft angle of attack and air speed detection by redundant strip pressure sensors, in: Proceedings of IEEE Sensors 2004, Vienna, AT, 2004.

[10] S. Callegari, A. Golfarelli, M. Zagnoni, M. Tartagni, A. Talamelli, P. Proli, Experiments on aircraft flight parameter detection by on-skin sensors, in: Book of Extended Abstracts of Eurosensors 2005, Barcelona, SP, 2005. 
[11] DSA 3217/3218 Digital Sensor Array (data sheet G511), Scanivalve Corporation, 2003, available at http://www.scanivalve.com.

[12] N.P. Kim, M.J. Holland, M.H. Tanielian, R. Poff, MEMS sensor multichip module assembly with $\mathrm{TAB}$ carrier-pressure belt for aircraft flight testing, in: Proceedings of the Electronic Components and Technology Conference, 2000, pp. 689-696.

[13] S.P. Timoshenko, S. Woinowky-Krieger, Theory of Plates and Shells, 2nd ed., McGraw-Hill, 1959.

[14] A. Golfarelli, M. Zagnoni, P. Proli, S. Callegari, A. Talamelli, E. Sangiorgi, M. Tartagni, Acquisition system for pressure sensor network, in: Proceedings of IEEE Sensors 2004, Vienna, AT, 2004.

[15] S.M. Kay, Fundamentals of statistical signal processing Estimation Theory, vol. 1, Prentice Hall, Upper Saddle River, NJ, 1993.

[16] H.A. Eiselt, G. Pederzoli, C.L. Sandblom, Continuous Optimization Models, de Gruyter, Berlin, 1987.

[17] M. Drela, XFOIL: an analysis and design system for low reynolds number airfoils, in: Proceedings of the Conference on Low Reynolds Number Airfoil Aerodynamics, University of Notre Dame, 1989.

[18] M. Drela, Y. Harold, XFOIL 6.94 User Guide, Aerospace Computational Design Lab (ACDL) Department of Aeronautics and Astronautics, Massachusetts Institute of Technology, 2001, online at http://raphael.mit.edu/xfoil.

\section{Biographies}

Sergio Callegari received the Dr Eng degree (with honors) in electronic engineering and the $\mathrm{PhD}$ degree in electronic engineering and computer science from the University of Bologna, Italy, in 1996 and 2000, respectively, working on the study of non-linear circuits and chaotic systems. In 1996, he was a visiting student at King's College London, London, UK. He is currently a researcher and assistant professor at the School of Engineering II, University of Bologna, where he teaches digital electronics and sensors. At the same university, he has also joined the recently instituted Advanced Research Center on Electronic Systems for Information and Communication Technologies. His current research interests include sensor design, non-linear signal processing, internally non-linear, externally linear networks, and chaotic maps. $\mathrm{He}$ has authored or co-authored more than 45 papers in international conferences, journals and scientific books. In 2004, he was co-recipient of the IEEE Circuit and Systems Society Darlington Award, for the best paper appeared in the IEEE Transactions on Circuits and Systems in the previous biennium.

Michele Zagnoni received the Dr Eng degree in electronic engineering in 2002 from the University of Bologna, Italy. In 2002, he also joined the Advanced Research Center for Electronic Systems for Information and Communication Technologies at the same university where he is now a $\mathrm{PhD}$ student in electronic engineering at the department of electronics and computer science. In 2005, he was a visiting student at the School of Electronic and Computer Science (ECS) at the Southampton University and at the Physics department of the University of Oxford. He is currently a researcher at the University of Southampton. His research interests have included: pressure sensors design, finite element method simulations, sorting algorithms, and impedance analysis. Currently he focuses on electrically addressable artificial lipid bi-layer arrays on chip and ion channels recording.

Alessandro Golfarelli received the degree in electronic engineering from the University of Bologna, Italy, Bologna, in 1999. From 2000 to 2002, he was with the "Laboratori Fondazione G. Marconi" of Pontecchio Marconi, Bologna, Italy, working as integrated circuit designer in MMIC and RFIC area. He is currently a collaborator with the Advanced Research Center on Electronic Systems for Information and Communication Technologies at the University of Bologna. His research focuses on acquisition systems for sensor networks.

Marco Tartagni received the Dr Eng degree in electrical engineering in 1988 and the $\mathrm{PhD}$ degree in electrical engineering and computer sciences in 1993 from the University of Bologna, Italy. He joined the Department of Electrical Engineering of the California Institute of Technology, Pasadena, CA in 1992 and in 1994 as Research Fellow, working on various aspects of analog VLSI for image processing. Since 1995, he is with the Department of Electronics of the University of Bologna where he is currently associate professor. From 1996 to 2001, he has been a team leader in the joint lab between ST-Microelectronics and University of Bologna working on intelligent sensors such as CMOS cameras and the first silicon-only fingerprint capacitive sensor, now a commercial product. Since 2001, he is with the Advanced Research Center on Electronic Systems for Information and Communication Technologies, University of Bologna working on sensor networks, hybrid technologies for cellular and molecular biology and impedance characterization. $\mathrm{He}$ is also interested in analog design and in the sensing limits of microelectronic sensors. He is co-founder of Silicon Biosystems, s.r.l. and co-recipient of the Van Vessem Outstanding Paper Award at the 2004 IEEE International Solid State Conference.

Alessandro Talamelli graduated in aeronautical engineering (with honors) at the University of Pisa in 1990. He became a $\mathrm{PhD}$ in aerospace engineering at Politec-nico di Milano, 1994. Researcher in fluid mechanics at Università of Pisa from 1995, he became associate professor in aerodynamics at the University of Bologna in 2001. Since 2003, he is guest lecturer at the Royal Institute of Technology KTH in Stockholm and associate lecturer at the University of Modena. His main research activities focus on fluid mechanics, in particular experimental studies of turbulence in jets and confined flows. $\mathrm{He}$ is co-author of more than 40 papers in journals and conferences.

Paolo Proli received the first level degree in mechanical engineering from the University of Bologna, Italy, Bologna, in 1999. Since 1998, he is a technician of the Aerospace Engineering Laboratories of the same University, where he is in charge of the Fluid Mechanics Facilities.

Alessandro Rossetti received the Dr Eng degree in aerospace engineering from the University of Bologna, Italy in 2004. He is currently attending the first year of a $\mathrm{PhD}$ course in fluid-dynamics, at the same institution. He is also a collaborator with the Advanced Research Center on Electronic Systems for Information and Communication Technologies, at the University of Bologna. His research focuses on turbulence, wall bounded turbulent flows, and design of array-type pressure sensors for airplane wings and sail planes. 